\title{
Inflammatory parameters in elderly women with and without sarcopenic obesity
}

\author{
Silvana Schwerz Funghetto (iD) 1, Alessandro de Oliveira Silva (D) 2, Maurílio Tiradentes \\ Dutra (iD 3*, Marina Morato Stival1, Yuri Gustavo de Sousa Barbalho1, Mateus Medeiros \\ Leite1, Marcio Rabelo Mota ${ }^{2}$, Luciano Ramos de Lima ${ }^{1}$, Izabel Cristina Rodrigues da \\ Silva ${ }^{1}$ \\ ${ }^{1}$ Graduate Program in Health Sciences and Technologies, Faculty of Ceilandia, University of Brasilia, Federal District, \\ Brasília, Brazil. \\ ${ }^{2}$ University Center of Brasilia - UniCEUB, Brasilia, DF, Brazil. \\ ${ }^{3}$ Federal Institute of Education, Science and Technology of Brasília. Campus Estrutural, Área Especial nº 01, Quadra 16, \\ Cidade do Automóvel/SCIA, 71250-000, Cidade Estrutural, DF, Brazil. \\ *Corresponding author. E-mail: maurilio.dutra@ifb.edu.br
}

\begin{abstract}
The combination of increased fat mass with a decrease in muscle mass and strength in the elderly has been termed sarcopenic obesity (SO). In addition, augmented levels of inflammatory markers have been reported in subjects with SO. Two hundred-sixteen obese elderly women were included in this crosssectional study. They underwent body composition analysis by DEXA to define the presence of SO. Volunteers were divided into two groups: $\mathrm{SO}(\mathrm{n}=83 ; 66.7 \pm 5.6$ years) and non-SO $(\mathrm{n}=133 ; 67.6 \pm 4.9$ years) for the comparison of inflammatory cytokines. There were no significant differences between $\mathrm{SO}$ and non-SO groups in the blood concentrations of the inflammatory markers analyzed, e.g. interleukin-6 $(0.82 \pm 0.20 \mathrm{vs}$ $0.83 \pm 0.19 \mathrm{pg} / \mathrm{ml} ; \mathrm{p}=0.64)$, C-reactive protein $(2.70 \pm 1.55 \mathrm{vs} 2.82 \pm 1.66 \mathrm{pg} / \mathrm{ml} ; \mathrm{p}=0.71)$, tumor necrosis factor alpha $(0.71 \pm 0.08$ vs $0.70 \pm 0.08 \mathrm{pg} / \mathrm{ml} ; \mathrm{p}=0.42)$ and interferon-gamma $(0.75 \pm 0.14$ vs $0.74 \pm 0.08 \mathrm{pg} / \mathrm{ml}$; $\mathrm{p}=0.47)$, respectively. Thus, in the studied population, inflammatory markers are not exacerbated by SO when compared to obesity without sarcopenia.
\end{abstract}

Keywords: Inflammation, Obesity, Sarcopenia, Aging, Body composition.

DOI: https://doi.org/10.33837/msj.v3i3.1306

Received August 27, 2020. Accepted September 22, 2020.

Associate editor: André Gadelha

\section{INTRODUCTION}

Increased longevity is a widespread global trend as evidenced by demographic and epidemiological data, especially regarding females, who live an average of seven years longer than males (Cullen et al., 2016). According to data from the Brazilian Institute of Geography and Statistics, there will be an increase in the population of people aged 60 years and older from $10 \%$ in 2010 to $29,3 \%$ in 2050 (Simões, 2016).

With an aging population comes a higher prevalence of chronic conditions such as cardiovascular disease, obesity, cancer, and diabetes (Prince et al., 2015). Moreover, the decrease in muscle mass and strength combined with the increase in fat mass contribute to the loss of functional capacity related to aging (Papa et al., 2017). Previous data has already shown that obesity is associated with chronic

Copyright $(0)$ The Author(s).

This is an open-access paper published by the Instituto Federal Goiano, Urutai - GO, Brazil. All rights reserved. It is distributed under the terms of the Creative Commons Attribution 4.0 International License. inflammation and this is evidenced by an increase in blood inflammatory markers such as, interleukin-6 (IL6), tumor necrosis factor-alpha (TNF- $\alpha)$, interleukin 1 beta (IL-1 $\beta$ ) and C-reactive protein (CRP) (Bano et al., 2017, Yang et al., 2015). Of note, IL-6 is a cytokine produced by several cell types and mediate acute phase inflammation. TNF-a is related to cytolysis, especially in tumoral cells. However, high concentration of this marker is related to worst prognosis in cancer and inflammatory shock (Forte, 2007).

Muscle mass usually comprises $50 \%$ of total body weight in young adults. However, it decreases by up to $25 \%$ in individuals 75 to 80 years old (Buford et al., 2010, Sakuma et al., 2014). Post-menopausal women present an accelerated loss of muscle mass and strength compared with men of similar age (Chen et al., 2017). This process of losing muscle mass and strength (e.g. sarcopenia) might be related to hormonal changes, sedentary lifestyle, poor diet, inflammatory markers, products of oxidative damage, serum creatinine and urinary creatinine excretion, endocrine function, urine proteomics panel, N-terminal procollagen peptides, myostatin and agrin fragment 
(Fougère et al., 2015). These negative outcomes suggest that sarcopenia and obesity have independent and additive adverse effects on health and that the efforts taken to understand the aging process should focus on obesity and maintaining or increasing skeletal muscle mass and function.

The loss of muscle mass is usually accompanied by a concomitant increase in fat mass. The combination of increased fat mass with a decrease in muscle mass and strength in the elderly has been termed sarcopenic obesity (SO) (Cruz-Jentoft et al., 2010, Narici and Maffulli, 2010). This pathological condition is associated with functional limitations and increased mortality (Batsis et al., 2015). Nevertheless, elderly obese women with and without SO present several abnormalities in skeletal muscle. Particularly, intramuscular lipid accumulation, which has been associated with low force generation, insulin resistance, reduced regenerative capacity from injuries, resistance to anabolic stimulus (growth factors, hormones, amino acids and exercise) and heightened local inflammatory pathways (Hamrick et al., 2016).

Augmented levels of inflammatory cytokines have been reported in subjects with SO (Wilson et al., 2017). These markers of inflammation produced by adipose tissue and inflammatory immune cells may accelerate the rate of muscle breakdown, reducing muscle strength and contributing to the development of sarcopenia and SO (Dutra et al., 2017). In this regard, studies have shown that elderly women with SO have greater functional limitations, lower muscle strength and increased risk of death compared with non-sarcopenic obese older adults (Lustosa et al., 2017, Silva Neto et al., 2012). Furthermore, research to date have focused on older people with obesity, fragility, or post-menopausal women separately and not with the SO condition (Prestes et al., 2009).

Therefore, the aim of this study was to compare inflammatory blood markers of elderly obese women with and without SO. Initially, the hypothesis of the present investigation was that elderly women with SO would present higher concentrations of inflammatory markers compared with elderly obese women without SO.

\section{METHODS}

\section{Sample}

Two hundred-sixteen obese elderly women participated in this cross-sectional study. Volunteers were recruited from a community in the Federal District of Brazil. They were divided into two groups: SO $(\mathrm{n}=83 ; 66.7 \pm 5.6$ years $)$ and non-SO $(\mathrm{n}=133 ; 67.6$ \pm 4.9 years). A questionnaire was applied to measure the level of physical activity (International Physical Activity Questionnaire - IPAQ) and only sedentary women (aged 60 years or more) who underwent anthropometric and body composition analysis were selected to participate. Subjects with autoimmune or rheumatic diseases, as well as those who were taking medications (such as beta-blockers and/or metformin) were excluded from the study. The volunteers were not part of any hormone therapy program.

Enrollment was voluntary and written consent was obtained from each subject. The methodology of the present study was approved by the Ethics Committee for Human Research of the Catholic University of Brasília (process number 235/2010) and the procedures were conducted in accordance with guidelines for experimentation with human subject.

\section{Body Composition, obesity and sarcopenic obesity}

All selected volunteers underwent body composition analysis by Dual-energy X-ray absorptiometry (DXA) (General Electric-GE model 8548 BX1L, Lunar DPX type, software Encore 2005; Rommelsdorf, Germany). Appendicular fat free mass (AFFM) was calculated as the sum of the fat free mass (FFM) from both arms and legs obtained by DXA.

Sarcopenic obesity was defined according to criteria proposed by Oliveira et al. (2011). Briefly, the method is based on the residual values of a regression equation that predicts appendicular FFM based on height (in meters) and fat mass (in $\mathrm{kg}$ ). The equation for elderly women has been identified as follows: predicted AFFM = $-14.529+(17.989$ * Height in meters $)$ $+(0.1307$ * total fat mass in $\mathrm{kg})$. The residual value (i.e., DXA measured AFFM - equation predicted AFFM) was used for classification of SO, with a cutoff value of $\leq 3.4$.

Obesity was determined by DXA and by body mass index (BMI). The reference values for body fat percentage followed the recommendations of the National Institute of Diabetes and Digestive and Kidney Diseases (Eckel et al., 2002), with a cutoff value of $32 \%$ for women. Obesity determined by BMI followed the proposal of Lipschitz (Lipschitz, 1994), with a cutoff value of $27.0 \mathrm{~kg} / \mathrm{m}^{2}$ for elderly women.

\section{Inflammatory parameters}

The following inflammatory markers were measured: IL-6, TNF-a, interferon-gama (INF-Y) and CRP. The cytokine assay was performed using the ELISA (enzyme-linked immunosorbent assay) method according to the manufacturer ( $R$ \& D Systems, Minneapolis, MN, USA). Plasma CRP was determined by turbidimetry according to the manufacturers' protocol (Roche Diagnostic, GmBH - Germany).

All measurements were performed in triplicate and the mean values were reported in the present study. The classification described by Harris et al. (1999) was adopted, in which, values $\geq 2.08 \mathrm{pg} / \mathrm{ml}$ 
were considered to be high for IL-6 and $\geq 2.78 \mathrm{pg} / \mathrm{mL}$ for CRP.

Regarding TNF-a, concentrations up to 4.12 $\mathrm{pg} / \mathrm{ml}$ were considered to be normal, as described by the manufacturer. Finally, the levels of INF- $\gamma$ were presented as the mean and percentage in order to compare this marker with the aforementioned inflammatory markers, as well as with previous studies that have measured the serum levels of this cytokine.

\section{Statistical Analysis}

Data are presented as mean \pm standard deviation (SD) and percentages when appropriate. Data normality was tested by the Kolmogorov-Smirnov test. The inflammatory parameters did not present normal distribution, thus values were natural-log transformed. The Student t-test for independent samples was applied to determine the differences between groups (SO vs non-SO). Pearson's correlation test was used to examine the relationship of inflammatory markers with age, body mass index, body fat mass, and fat-free mass. Sample size was determined a priori using G*Power version 3.1.3 (Kiel, Germany) with the level of significance set at $\mathrm{p}=0.05$ and power $(1-\beta)=0.80$. We conducted a pilot study to evaluate the effect size for each inflammatory variable and the mean effect size d was 0.34 (small to medium effect size). Based on these a priori calculations and the pilot study, we set the final sample size. The level of significance was set at $\mathrm{p}<0.05$. The statistical software used was the SPSS version 21.0 (IBM Corporation, Armonk, NY, USA).

\section{RESULTS}

The mean age of all the subjects of the present study was $67.2 \pm 5.1$ years. Anthropometric characteristics of the groups according to $\mathrm{SO}$ classifications are presented in Table 1. From all subjects, 38.4\% $(\mathrm{n}=83)$ was classified with SO, while $61.6 \%(n=133)$ were not. However, all volunteers were obese according to their body fat percentage (Baumgartner et al., 1998). Sarcopenic obese subjects presented mean values of appendicular lean mass (ALM) measured by DXA similar to those who were not classified with SO. As expected, FFM was significantly higher $(p=0.001)$ for the group without $\mathrm{SO}$ as compared with the $\mathrm{SO}$ group.

Table 1. Anthropometric variables.

\begin{tabular}{ccccc} 
& $\begin{array}{c}\text { SO } \\
(\mathrm{n}=83)\end{array}$ & $\begin{array}{c}\text { Non-SO } \\
(\mathrm{n}=133)\end{array}$ & $\mathrm{P}$ & $\begin{array}{c}95 \% \mathrm{CI} \\
\text { of the difference }\end{array}$ \\
\cline { 2 - 5 } Age (years) & $66.7 \pm 5.6$ & $67.6 \pm 4.9$ & 0.23 & $(-2.30 / 0.55)$ \\
TBM (kg) & $68.1 \pm 8.0$ & $65.4 \pm 7.4$ & 0.42 & $(-1.28 / 3.02)$ \\
Heigth (cm) & $1.55 \pm 0.05$ & $1.50 \pm 0.05^{*}$ & 0.001 & $(0.04 / 0.07)$ \\
BMI (kg/m $\left.{ }^{2}\right)$ & $28.1 \pm 2.9$ & $29.9 \pm 3.5^{*}$ & 0.001 & $(-2.64 /-0.82)$ \\
BF (\%) & $40.5 \pm 3.9$ & $41.3 \pm 3.5$ & 0.13 & $(-1.80 / 0.23)$ \\
BF (kg) & $26.7 \pm 7.7$ & $23.2 \pm 7.1^{*}$ & 0.001 & $(1.51 / 5.55)$ \\
FFM (kg) & $12.3 \pm 1.4$ & $14.4 \pm 1.3^{*}$ & 0.001 & $(-2.48 /-1.74)$ \\
\hline
\end{tabular}

Values are presented as means \pm DP; BMI: body mass index; BF: body fat. TBM: total body mass; SO: sarcopenic obesity; FFM: fat free mass; CI: confidence interval. ${ }^{*}$ Statistically different between groups: $\mathrm{p} \leq 0.05$.

Regarding the inflammatory profile, there were no statistically significant differences between groups (Table 2). Moreover, the values of TNF-a and INF- $\gamma$ were found to be normal for all subjects. However, $55 \%$ of the sample presented levels of CRP above $0.8 \mathrm{pg} / \mathrm{mL}$ and at least $26 \%$ of them had very high CRP levels. In addition, IL-6 levels were high in $57 \%$ of the sample.

Table 2. Inflammatory parameters according to sarcopenic obesity classification. Values are natural-log transformed.

\begin{tabular}{ccccc}
\hline & $\mathrm{SO}(\mathrm{n}=83)$ & $\begin{array}{c}\text { Non-SO } \\
(\mathrm{n}=133)\end{array}$ & $\mathrm{P}$ & $\begin{array}{c}95 \% \mathrm{CI} \\
\text { of the } \\
\text { difference }\end{array}$ \\
\cline { 2 - 5 } $\begin{array}{c}\mathrm{IL}-6 \\
(\mathrm{pg} / \mathrm{mL})\end{array}$ & $0.59 \pm 0.09$ & $0.60 \pm 0.09$ & 0.58 & $(-0.03 / 0.02)$ \\
$\begin{array}{c}\mathrm{CRP} \\
(\mathrm{pg} / \mathrm{mL})\end{array}$ & $1.23 \pm 0.41$ & $1.25 \pm 0.43$ & 0.79 & $(-0.18 / 0.14)$ \\
$\begin{array}{c}\mathrm{TNF}-\alpha \\
(\mathrm{pg} / \mathrm{mL})\end{array}$ & $0.53 \pm 0.05$ & $0.53 \pm 0.04$ & 0.40 & $(-0.01 / 0.02)$ \\
$\begin{array}{c}\mathrm{INF}-\gamma \\
(\mathrm{pg} / \mathrm{mL})\end{array}$ & $0.55 \pm 0.07$ & $0.55 \pm 0.04$ & 0.56 & $(-0.01 / 0.02)$ \\
\hline
\end{tabular}

SO: sarcopenic obesity; IL-6: interleukin-6; CRP: C-reactive protein; TNF-a: tumor necrosis factor alpha; INF- $\gamma$ : interferon gamma.

The relationship between inflammatory variables with age, body mass index, body fat mass and fat-free mass is presented in table 3. TNF-a was positively correlated with age $(\mathrm{r}=0.16, \mathrm{p}<.05)$, and negatively correlated with body mass index $(r=-0.19$, $\mathrm{p}<.05)$. Inflammatory variables (IL-6, CRP and INF- $\gamma$ ) did not correlate with age, nor with anthropometric variables. Of note, both IL-6 $(\mathrm{r}=0.36, \mathrm{p}<.05)$ and TNF- $\mathrm{a}(\mathrm{r}=0.18, \mathrm{p}<.05)$ presented a significant correlation with INF- $\gamma$.

Table 3. Coefficients of correlation between inflammatory markers, age, body fat, and fat-free mass $(\mathrm{n}=216)$.

\begin{tabular}{ccccc} 
& Age & BMI & Body Fat & FFM \\
IL-6 & 0.02 & 0.02 & -0.06 & -0.06 \\
CRP & -0.07 & -0.16 & -0.04 & 0.06 \\
TNF-a & $0.16^{*}$ & $-0.19^{*}$ & 0.11 & 0.00 \\
INF- $\gamma$ & -0.01 & -0.02 & 0.02 & -0.12 \\
\hline
\end{tabular}

IL-6: interleukin-6; CRP: C-reactive protein; TNF-a: tumor necrosis factor alpha; INF- $\gamma$ : interferon gamma; BF: body fat; FFM: fat-free mass. ${ }^{*} \mathrm{p}<.05$.

\section{DISCUSSION}

The aim of this study was to compare inflammatory markers in elderly obese women with and without SO. Contrary to the initial hypothesis, obese elderly women with SO did not present higher concentrations of inflammatory markers as compared to those without SO. Moreover, TNF-a was positively correlated with age, and negatively correlated with body mass index. Both IL-6 and TNF-a were correlated with INF-y.

A high prevalence of SO (38.4\%) was observed in the present study compared to previous published articles, where the prevalence ranged from $3 \%$ to 
$17.7 \%$ (Baumgartner, 2000). Similar to the results of the present investigation, a study conducted in New Mexico, observed an even higher prevalence of SO (48.1\%) (Baumgartner et al., 1998). On the other hand, Oliveira et al. (2011) reported a prevalence of $19.8 \%$ in Brazilian elderly women. It is noteworthy that all subjects investigated in this study were obese according to their body fat percentage, which may have elevated the prevalence of SO.

Epidemiological evidence revealed that $\mathrm{SO}$ is associated with functional decline (Villareal et al., 2011) and the risk of developing cardiovascular, metabolic and endocrine diseases, denervation and mitochondrial dysfunction (Sakuma and Yamaguchi, 2013). Lee et al. (2016) demonstrated that SO individuals were more likely $(\mathrm{OR}=3.51)$ to develop knee osteoarthritis as compared with those who were not sarcopenic obese, although both groups had the same body mass. Previous studies revealed that IL-6, $\mathrm{CRP}$, and other cytokines can lead to the development of insulin resistance (IR). It is known that IR is associated with sarcopenia (Kalyani, Corriere, Ferrucci 2014) and cognitive performance, while no differences were evidenced between elderly with and without OS (Levine and Crimmins, 2012).

Yang et al.(2015) analyzed the impact of sarcopenic obesity on inflammatory markers, including interleukin-6 (IL-6), high-sensitivity Creactive protein (hs-CRP), and tumor necrosis factoralpha (TNF-a) of 844 community-dwelling people aged 65 years and older. The authors demonstrated the significant interaction between sex and sarcopenic obesity status in serum hs-CRP levels, as evidenced by higher hs-CRP levels among males in the obesity only and in the sarcopenic obesity groups compared with normal males. This is probably related to body fat mediated inflammation, once men usually present more fat in the central region in the body (i.e. visceral fat), which is strongly related to metabolic disturbances.

The results of the present study revealed a similar inflammatory profile between elderly obese women with and without SO (Table 2). These results can be partially explained since adipose tissue is a major contributor to the production of proinflammatory cytokines, such as TNF-a and IL-6 (Jensen and Hsiao, 2010). Thus, it seems that muscle mass can be directly affected by inflammation, and elevated levels of IL-6 directly affect anabolic efficacy of IGF-I, which is a likely mechanism that contributes to the progressive decrease of lean mass in the elderly (Kalyani, Corriere, Ferrucci, 2014). In this regard, IL-6, TNF-a, and CRPare also reported to play negative effects on physical function, which are thought to accelerate age-associated changes in body composition. Yet, it is noteworthy that Dutra et al. (2017) found that IL-6 was significantly higher in SO elderly women when compared to non-SO, which is different from the present investigation. These discrepancies may be related to two associated circumstances. First, sample size of SO women in the study by Dutra et al. was only a third of the present sample size. Moreover, cytokines concentration may vary significantly even among samples of similar characteristics and they rarely present to be normally distributed.

According to Singh and Newman (2011), the high blood concentration of CRP, which is also an acute phase marker, has been extensively associated with the prediction of cardiovascular diseases, as well as with a marked physical decline in the elderly (Penninx et al., 2004). Similarly, Schaap et al. (2009) demonstrated that elevated blood concentrations of CRP were associated with approximately $40 \%$ loss of muscle strength after a follow-up of 3 years. In the present study, a high percentage of volunteers (55\%) presented levels of CRP considered to be above the recommended levels for cardiovascular health. Furthermore, Stenholm et al. (2008) found that the combination of high body fat percentage and low hand grip strength was associated with increased levels of CRP. A previous study examining an Asian population demonstrated that high-sensitivity (hs)CRP levels were significantly and independently associated with SO, even after adjusting for several other risk factors (Kim et al., 2013). Therefore, lowgrade inflammation might be one of the principal factors involved in the vicious cycle of sarcopenia and obesity in the elderly.

Some methodological limitations of this study should be highlighted. Firstly, it was a cross-sectional design study with a relatively small number of subjects, according to the small sample power (even with the a priori power analysis), making it impossible to establish a cause and effect relationship between $\mathrm{SO}$ and the inflammatory response in the long-term. We also understand that there may be a selection bias in the present study. However, the number of subjects enrolled in the study was significant due to time and cost consuming aspects related to DXA and inflammatory markers analyses. Unfortunately, cytokines tend to present a wide variability in humans, which directly affects the sample power analysis. To compensate that, subjects were limited to women, removing the potential influence of gender on the results.

Secondly, individuals from both groups were obese according to the percentage of body fat and the study does not have a control group. This fact limits the discussion of the present results, since it is not possible to infer if subjects without SO and without regular obesity (i.e. obesity without sarcopenia) would present a different inflammatory profile compared to those with SO and regular obesity. Finally, the definition of sarcopenia proposed by the European Working Group on Sarcopenia in Older People 
(EWGSOP), which recommends the evaluation of muscle strength for the diagnosis of sarcopenia was not used in this study (Cruz-Jentoft et al., 2010).

\section{CONCLUSION}

In conclusion, the results of this study demonstrated that blood inflammatory markers were not exacerbated by $\mathrm{SO}$ in the studied population. In addition, both groups presented a high inflammatory profile, demonstrating that obesity per se may explain a greater likelihood of inflammation in elderly women.

\section{ACKNOWLEDGEMENTS}

This work was carried out with support from the Higher Education Personnel Improvement Coordination, Brazil (CAPES) (Financing Code 001). This study was funded by the Federal District Research Support Foundation (FAP/DF) (promotion notice 03/2016), the National Council for Scientific and Technological Development (CNPq) (421836/2016-4) and University of Brasilia.

\section{CONFLICT OF INTEREST}

The authors declare no conflict of interest.

\section{REFERENCES}

Bano, G., Trevisan, C., Carraro, S., Solmi, M., Luchini, C., Stubbs, B., et al. (2017). Inflammation and sarcopenia: A systematic review and meta-analysis. Maturitas 96, 10-15. doi:10.1016/j.maturitas.2016.11.006.

Batsis, J. A., Mackenzie, T. A., Lopez-Jimenez, F., and Bartels, S. J. (2015). Sarcopenia, sarcopenic obesity, and functional impairments in older adults: National Health and Nutrition Examination Surveys 1999-2004. Nutr. Res, 35(12), 1031-1039. doi:10.1016/j.nutres.2015.09.003.

Baumgartner, R. N. (2000). Body composition in healthy aging. Ann N Y Acad Sci, 904, 437-448. doi:10.1111/j.17496632.2000.tb06498.x.

Baumgartner, R. N., Koehler, K. M., Gallagher, D., Romero, L., Heymsfield, S. B., Ross, R. R., et al. (1998). Epidemiology of Sarcopenia among the Elderly in New Mexico. Am. J. Epidemiol. 147, 755-763. doi:10.1093/oxfordjournals.aje.a009520.

Buford, T. W., Cooke, M. B., Manini, T. M., Leeuwenburgh, C., and Willoughby, D. S. (2010). Effects of age and sedentary lifestyle on skeletal muscle NF-kappaB signaling in men. J. Gerontol. A. Biol. Sci. Med. Sci., 65(5), 532-537. doi:10.1093/gerona/glp196.

Chen, L., Xia, J., Xu, Z., Chen, Y., and Yang, Y. (2017). Evaluation of Sarcopenia in Elderly Women of China. Int. J. Gerontol. 11, 149-153.doi: 10.1016/j.ijge.2016.04.005.

Cruz-Jentoft, A. J., Baeyens, J. P., Bauer, J. M., Boirie, Y., Cederholm, T., Landi, F., et al. (2010). Sarcopenia: European consensus on definition and diagnosis. Age Ageing 39(4), 412-423. doi:10.1093/ageing/afq034.

Cullen, M. R., Baiocchi, M., Eggleston, K., Loftus, P., and Fuchs, V. (2016). The weaker sex? Vulnerable men and women's resilience to socio-economic disadvantage. SSM - Popul. Heal. 2, 512-524. doi:10.1016/j.ssmph.2016.06.006.

Dutra, M. T., Avelar, B. P., Souza, V. C., Bottaro, M., Oliveira, R. J., Nóbrega, O. T., et al. (2017). Relationship between sarcopenic obesity-related phenotypes and inflammatory markers in postmenopausal women. Clin. Physiol. Funct. Imaging 37(2), 205-210. doi:10.1111/cpf.12287.

Eckel, R. H., Barouch, W. W., and Ershow, A. G. (2002). Report of the National Heart, Lung, and Blood Institute-National Institute of Diabetes and Digestive and Kidney Diseases working group on the pathophysiology of obesity-associated cardiovascular disease. Circulation 105(24), 2923-2928. doi:10.1161/01.CIR.0000017823.53114.4C.

Forte, WCN. (2007). Imunologia: do básico ao aplicado. $2^{\mathrm{a}} \mathrm{ed}$. Porto Alegre: Artmed.

Fougère, B., Vellas, B., Van Kan, G. A., and Cesari, M. (2015). Identification of biological markers for better characterization of older subjects with physical frailty and sarcopenia. Transl. Neurosci. 6(1), 103-110. doi:10.1515/tnsci-2015-0009.

Hamrick MW, McGee-Lawrence ME, Frechette DM. (2016). Fatty Infiltration of Skeletal Muscle: Mechanisms and Comparisons with Bone Marrow Adiposity. Front. Endocrinol. (Lausanne). 7:69.doi: 10.3389 /fendo.2016.00069.

Simões CCS. (2016). Relações entre as alterações históricas na dinâmica demográfica brasileira e os impactos decorrentes do processo de envelhecimento da população. Rio de Janeiro: IBGE, Coordenação de População e Indicadores Sociais.

Jensen, G. L., and Hsiao, P. Y. (2010). Obesity in older adults: relationship to functional limitation. Curr. Opin. Clin. Nutr. Metab.Care 13(1), 46-51. doi:10.1097/ MCO.0b013e32833309cf.

Kim TN, Park MS, Lim KI, et al. (2013). Relationships between sarcopenic obesity and insulin resistance, inflammation, and vitamin D status: the Korean Sarcopenic Obesity Study. Clin. Endocrinol. (Oxf), 78(4), 525-532. doi: 10.1111/j.13652265.2012.04433.x.

Lee, D.-C., Shook, R. P., Drenowatz, C., and Blair, S. N. (2016). Physical activity and sarcopenic obesity: definition, assessment, prevalence and mechanism. Futur. Sci. OA, 2(3), FSO127. doi:10.4155/fsoa-2016-0028.

Levine, M. E., and Crimmins, E. M. (2012). The Impact of Insulin Resistance and Inflammation on the Association Between Sarcopenic Obesity and Physical Functioning. Obesity 20(10), 2101-2106. doi:10.1038/oby.2012.20.

Lipschitz, D. (1994). Screening for nutritional status in the elderly. Prim. Care 21(1), 55-67.

Lustosa, L. P., Batista, P. P., Pereira, D. S., Pereira, L. S. M., Scianni, A., and Ribeiro-Samora, G. A. (2017). Comparison between parameters of muscle performance and inflammatory biomarkers of non-sarcopenic and sarcopenic elderly women. Clin. Interv. Aging 12, 1183-1191. doi:10.2147/CIA.S139579.

Oliveira, R. J., Bottaro, M., Júnior, J. T., Farinatti, P. T. V., Bezerra, L. A., and Lima, R. M. (2011). Identification of sarcopenic obesity in postmenopausal women: A cutoff proposal. Brazilian J. Med. Biol. Res., 44(11), 1171-1176. doi:10.1590/S0100-879X2011007500135.

Papa, E. V, Dong, X., and Hassan, M. (2017). Skeletal Muscle Function Deficits in the Elderly: Current Perspectives on Resistance Training. J. Nat. Sci., 3, 1-8. doi:10.1016/j.jaac.2013.12.025.

Prestes J, Shiguemoto G, Botero JP, Frollini A, Dias R, Leite R, et al. (2009). Effects of resistance training on resistin, leptin, cytokines, and muscle force in elderly post-menopausal women. J. Sports Sci., 27(14), 1607-15. doi: 10.1080/02640410903352923.

Prince, M. J., Wu, F., Guo, Y., Gutierrez Robledo, L. M., O’Donnell, M., Sullivan, R., et al. (2015). The burden of disease in older people and implications for health policy and practice. Lancet 385(9967), 549-562. doi:10.1016/S0140-6736(14)61347-7.

Sakuma, K., Aoi, W., and Yamaguchi, A. (2014). The intriguing regulators of muscle mass in sarcopenia and muscular dystrophy. Front. Aging Neurosci. 6, 230. doi:10.3389/fnagi.2014.00230.

Sakuma, K., and Yamaguchi, A. (2013). Sarcopenic obesity and endocrinal adaptation with age. Int. J. Endocrinol. 2013. doi:10.1155/2013/204164

Silva Neto, L. S., Karnikowiski, M. G. O., Tavares, A. B., and Lima, R. M. (2012). Associação entre sarcopenia, obesidade sarcopênica e força muscular com variáveis relacionadas de 
qualidade de vida em idosas. Brazilian J. Phys. Ther. 16(5), 360-367. doi:10.1590/S1413-35552012005000044.

Singh, T., and Newman, A. B. (2011). Inflammatory markers in population studies of aging. Ageing Res. Rev. 10(3), 319-329. doi:10.1016/j.arr.2010.11.002

Stenholm S, Rantanen T, Heliövaara M, et al.(2008). The mediating role of C-reactive protein and handgrip strength between obesity and walking limitation. J. Am. Geriatr.Soc. 56(3), 462469. doi: 10.1111/j.1532-5415.2007.01567.x.

Villareal, D. T., Chode, S., Parimi, N., Sinacore, D. R., Hilton, T., Armamento-Villareal, R., et al. (2011). Weight loss, exercise, or both and physical function in obese older adults. N. Engl. J. Med. 364(13), 1218-29. doi:10.1056/NEJMoa1008234.

Wilson, D., Jackson, T., Sapey, E., and Lord, J. M. (2017). Frailty and sarcopenia: The potential role of an aged immune system. Ageing Res. Rev., 36, 1-10. doi:10.1016/j.arr.2017.01.006.

Yang, C. W., Li, C. I., Li, T. C., Liu, C. S., Lin, C. H., Lin, W. Y., et al. (2015). Association of sarcopenic obesity with higher serum high-sensitivity c-reactive protein levels in Chinese older males - A community-based study (Taichung Community Health Study-Elderly, TCHS-E). PLoS One 10 (8): e0136069. doi:10.1371/journal.pone.0132908.

To cite this paper:

Funghetto, S. S., Silva, A. O., Dutra, M. T., Stival, M. M., Barbalho, Y. G. S., Leite, M. M., Mota, M. R., Lima, L. R., Silva, I. C. R. (2020). Inflammatory parameters in elderly women with and without sarcopenic obesity. Multi-Science Journal, 3(3): 23-28. DOI: https:// doi.org/10.33837/msj.v3i3.1306 